\title{
Melanoma metastasis to a nonfunctioning pituitary macroadenoma: illustrative case
}

\author{
Krista Lamorie-Foote, BA, ${ }^{1}$ Shivani D. Rangwala, MD, ${ }^{1}$ Alexandra Kammen, MD, ${ }^{1}$ Esteban Gnass, MD, ${ }^{2}$ Daniel R. Kramer, MD, ${ }^{3}$ \\ Martin Rutkowski, MD, ${ }^{4}$ Ben A. Strickland, MD, ${ }^{1}$ John D. Carmichael, MD, ${ }^{1}$ and Gabriel Zada, MD ${ }^{1}$ \\ Departments of ${ }^{1}$ Neurosurgery and ${ }^{2}$ Pathology, Keck School of Medicine of University of Southern California, Los Angeles, California; ${ }^{3}$ Department of Neurosurgery, \\ University of Colorado, Aurora, Colorado; and ${ }^{4}$ Department of Neurosurgery, Augusta University Medical Center, Augusta, Georgia
}

\begin{abstract}
BACKGROUND Metastases to the central nervous system are often multiple in number and typically favor the gray-white matter junction. Collision tumors, defined as the coexistence of two morphologically different tumors, such as metastases to a known pituitary adenoma (PA), are exceedingly rare. Only a few reported cases of metastases to a PA exist in the literature.

OBSERVATIONS The authors present the case of a 64-year-old man with a known history of stage IV metastatic melanoma who was found to have hypermetabolic activity in the sellar region on surveillance positron emission tomography. On laboratory evaluation, he had clear evidence of pituitary axis dysfunction without diabetes insipidus. Subsequent magnetic resonance imaging showed a 2.4-cm sellar mass with features of a pituitary macroadenoma and internal hemorrhage, although no clinical symptoms of apoplexy were noted. He underwent a transsphenoidal endoscopic endonasal approach for resection of the sellar lesion. Final pathology showed a collision tumor with melanoma cells intermixed with PA cells.
\end{abstract}

LESSONS Histological analysis verified the rare presence of a collision tumor of a melanoma metastasis to a nonfunctional pituitary macroadenoma. Metastasis to a preexisting PA, although rare, should be considered in the differential diagnosis in patients with sellar lesions and a known cancer history.

https://thejns.org/doi/abs/10.3171/CASE2167

KEYWORDS melanoma; pituitary adenoma; metastasis; collision tumor; transsphenoidal craniotomy; endoscopic

Metastases to the pituitary gland are rare in the clinical setting, although patients with advanced malignancies have a high incidence of metastases on autopsy studies. ${ }^{1}$ Pituitary adenomas (PAs) are by far the most common sellar neoplasm. In a large case series, $90 \%$ of all patients undergoing transsphenoidal surgery for a sellar mass were diagnosed with a PA. ${ }^{2}$ Incidental intrasellar metastasis was diagnosed in only $4 \%$ of cases. ${ }^{3}$ Reported cases of pituitary metastases most commonly arise from primary malignancies in the lung and breast. ${ }^{4}$ Despite the propensity for melanoma to metastasize to the brain, there are few reported cases of metastasis to the sellar region. ${ }^{5-11}$ There are even fewer reported cases of a melanoma metastasis to a PA. ${ }^{12,13}$ Although subtle differences exist on magnetic resonance imaging (MRI) between PAs and melanoma based on the presence of melanin, which typically exhibits a T1 hyperintense signal versus a T1 isointense signal in PAs, ${ }^{14,15}$ the two pathologies remain difficult to differentiate on routine neuroimaging studies.

We present a rare case of a 64-year-old man with a history of diffusely metastatic acral lentiginous melanoma presenting with a sellar mass with characteristic imaging features of pituitary macroadenoma on MRI. After resection and pathological examination, final diagnosis revealed a collision tumor comprising melanoma metastasis to a nonfunctional PA.

\section{Illustrative Case}

A 64-year-old man was referred to the neurosurgery service at LAC + USC Medical Center in Los Angeles, California, after routine surveillance MRI had shown an enlarging sellar mass with new hemorrhage. The patient denied headache, visual disturbances, or any clinical symptoms of apoplexy and was neurologically intact at the time of initial neurosurgical evaluation. This patient had a wellknown history of diffusely metastatic acral lentiginous melanoma (pT4bN1b stage IIIC) involving the abdominal and pelvic lymph nodes, lung, and liver and was followed by oncology and endocrinology services for 2 years before referral to neurosurgery. On initial workup with the oncology department, the patient was found to

ABBREVIATIONS MRI = magnetic resonance imaging; PA = pituitary adenoma. INCLUDE WHEN CITING Published June 7, 2021; DOI: 10.3171/CASE2167.

SUBMITTED March 22, 2021. ACCEPTED March 29, 2021.

(C) 2021 The authors, CC BY-NC-ND 4.0 (http://creativecommons.org/licenses/by-nc-nd/4.0/). 

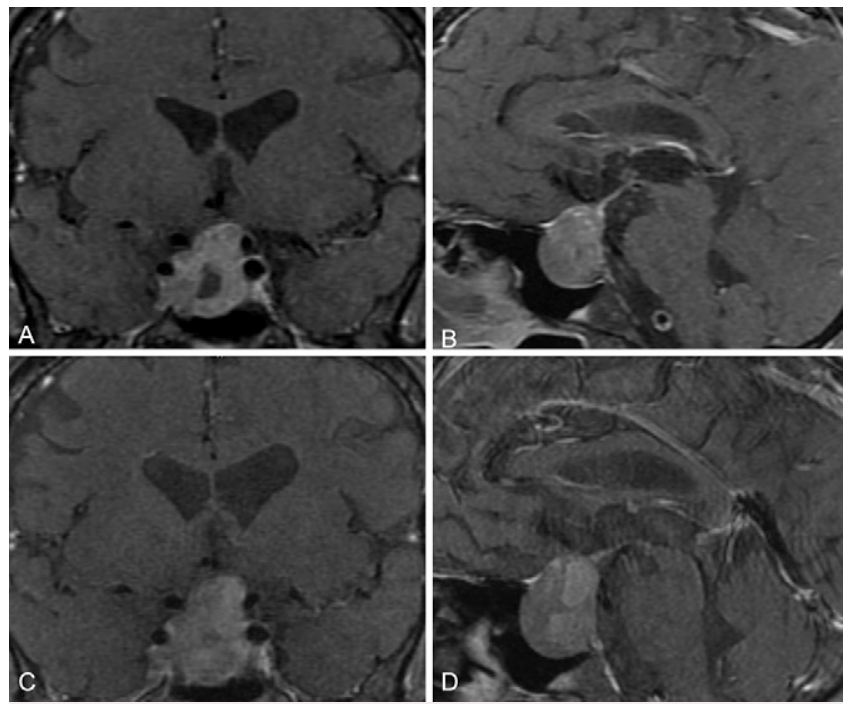

FIG. 1. Initial MRI shows a 2.4-cm heterogeneous contrast-enhancing sellar mass on coronal (A) and sagittal (B) views. Interval MRI shows enlargement of sellar mass and fluid-fluid levels suggestive of intratumoral hemorrhage on coronal (C) and sagittal (D) views.

have hypermetabolic activity on routine positron emission tomography imaging within the sellar region. Subsequent MRI showed a heterogeneously enhancing $2.4-\mathrm{cm}$ sellar mass (Fig. $1 \mathrm{~A}$ and B). He was referred to endocrinology after initial lab work showed decreased serum free T4, random cortisol, testosterone insulin-like growth factor-1, and mildly elevated prolactin indicative of hormonal axis dysfunction. At this initial referral, he did not have signs of diabetes insipidus, with normal serum sodium and no increased urinary symptoms. The endocrinology department started hormone replacement with levothyroxine and hydrocortisone, and the patient was serially monitored with annual $\mathrm{MRI}$, which was stable for 2 years. Ophthalmology service was not consulted initially because he did not report any visual symptoms and did not exhibit visual field deficits throughout the 2 years. During the 3rd year, MRI showed tumor progression with new internal hemorrhage (Fig. $1 \mathrm{C}$ and D), although the patient did not have any acute clinical symptoms, and he was referred to our neurosurgery service for further evaluation.

Because of concerns for tumor enlargement and new internal hemorrhage, the patient was scheduled for surgery. With a standard endoscopic endonasal transsphenoidal approach, the tumor was carefully resected and sent for permanent pathology. The tumor had a typical intraoperative appearance of a pituitary macroadenoma. The pituitary gland was identified and preserved, and all margins were believed to be free of tumor. All areas were inspected, and intraoperative gross-total resection was achieved. The arachnoid membrane remained intact with no evidence of an intraoperative cerebrospinal fluid leak. The patient had an uneventful recovery and remained neurologically intact. His 1-month follow-up with the endocrinology service demonstrated stable hypopituitarism on his regimen of levothyroxine and hydrocortisone, but low-dose testosterone was added to his hormone replacement regimen at that time.

Final pathology was consistent with two intermixed yet distinct tumor subtypes: a nonfunctioning PA with regions of metastatic melanoma (Fig. 2). PA cells stained positive for pan-cytokeratin and synaptophysin. The adenoma cells were negative for growth hormone, thyroid-stimulating hormone, adrenocorticotropic hormone, prolactin, T-pit, and Pit-1. The PA had diffuse nuclear staining for SF-1/NR5A1, which indicates a gonadotrophic PA. Melanoma cells stained positive for S100 and HMB45. The cells were noted to be intermixed throughout the specimen, suggesting a collision tumor of melanoma metastasis to a PA.

Oncology follow-up 3 months postoperatively showed disease progression of the melanoma with new bone metastasis on standard therapies (ibrutinib and pembrolizumab), with plans for enrollment in a phase II clinical trial combining talimogene laherparepvec (T-VEC) plus pembrolizumab for advanced metastatic melanoma. The patient died soon thereafter as the result of an unrelated accident and did not undergo standard 6-month postoperative MRI or enroll in a clinical study.

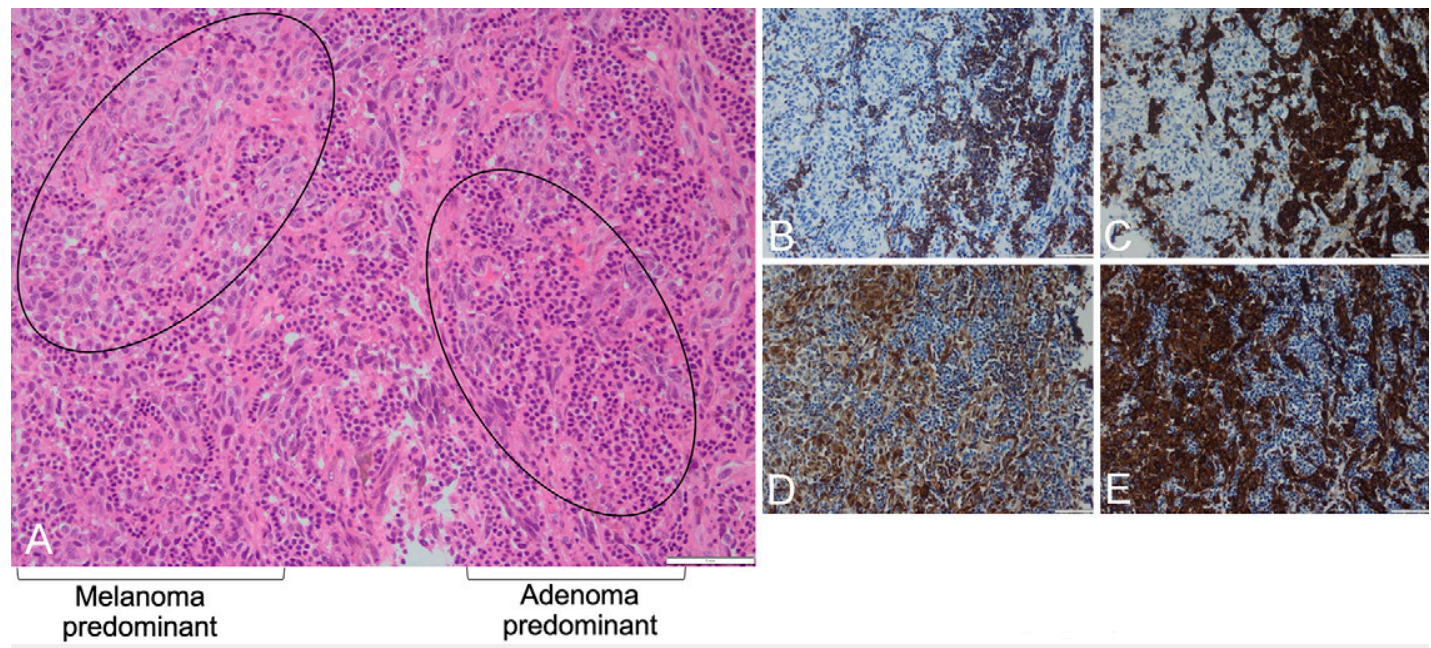

FIG. 2. A: Hematoxylin and eosin staining of tumor specimen area depicting both adenoma (right oval) and melanoma (left oval) and their respective immunohistochemical staining patterns. B and C: PA cells stained positive for adenoma markers pan-cytokeratin AE1/AE3 and synaptophysin. D and E: Melanoma cells stained positive for melanoma markers $\mathrm{S} 100$ and HMB45. Bars $=1 \mathrm{~mm}$. 
TABLE 1. Melanoma metastasis to PA

\begin{tabular}{|c|c|c|c|c|}
\hline Authors \& Yr & $\begin{array}{l}\text { Age }(Y r s) / \\
\quad \text { Sex }\end{array}$ & Primary Malignancy & Presentation & Notes \\
\hline Ramos et al., $2017^{13}$ & $67 / M$ & $\begin{array}{l}\text { Dorsal nodular } \\
\text { melanoma }\end{array}$ & $\begin{array}{l}\text { Suspected pituitary apoplexy: headache, } \\
\text { vomiting, decreased visual acuity }\end{array}$ & $\begin{array}{l}\text { History of pituitary macroadenoma } \\
\text { resection } \mathrm{w} / \text { radiotherapy }\end{array}$ \\
\hline Yang et al., $2017^{12}$ & $62 / F$ & Melanoma & $\begin{array}{l}\text { Progressive visual loss, headache, bilat } \\
\text { temporal hemianopsia, } \\
\text { hyperprolactinemia, elevated cortisol }\end{array}$ & $\begin{array}{l}\text { History of melanoma resection, } \\
\text { recurrence of melanoma \& } \\
\text { subsequent resection }\end{array}$ \\
\hline Jung et al., $2006^{25}$ & $70 / M$ & $\begin{array}{l}\text { Subungual acral } \\
\text { lentiginous } \\
\text { melanoma }\end{array}$ & $\begin{array}{c}\text { Suspected pituitary apoplexy: progressive } \\
\text { vision loss w/ modest hyperprolactinemia, } \\
\text { hypothyroidism, \& partial insufficiency of } \\
\text { adrenocorticotropic hormone }\end{array}$ & $\begin{array}{l}\text { History of metastatic malignant } \\
\text { melanoma }\end{array}$ \\
\hline
\end{tabular}

\section{Discussion \\ Observations}

We report a rare case of melanoma metastasis to a nonfunctioning pituitary macroadenoma. This phenomenon has been described in the literature as tumor-to-tumor metastases or collision tumor. Collision tumors typically are defined as neighboring neoplasms that invade each other. Tumor-to-tumor metastases define a broader category and are characterized by pathology demonstrating a ring of benign host tumor enclosing metastatic tumor and a known diagnosis of primary carcinoma with proven metastases. ${ }^{16}$ Meningiomas are the most common intracranial host tumors for metastasis, likely because of their increased vascular supply and common incidence among intracranial tumors. ${ }^{16-19}$ For similar reasons (e.g., because PAs are common sellar lesions and the pituitary gland is highly vascular), they are likely to create a favorable niche for metastatic tumor seeding and growth. Furthermore, absence of the blood-brain barrier (because PAs are extraarachnoidal tumors) may offer a further means of blood-borne tumor seeding within the vascular PA.

Pituitary metastases are a relatively uncommon occurrence in clinical practice. An autopsy study of patients with invasive carcinoma found that $1.8 \%$ had pituitary metastases. Of these, $83 \%$ were from a primary breast carcinoma and $11 \%$ were from a lung carcinoma. ${ }^{1}$ Although symptoms such as diabetes insipidus and hypopituitarism are more common in patients with metastases to the pituitary gland, ${ }^{4,9,20-22}$ most sellar metastases remain asymptomatic, ${ }^{4}$ unlike in our patient, who had known pituitary axis dysfunction. The prognosis for pituitary metastases is often poor because they frequently present during the final stages of malignancy., ${ }^{4,23} \mathrm{Al}$ though the occurrence of a metastasis to a PA is rare, given the frequency of PAs in the clinical setting, it should remain in the differential diagnosis. The capsular and inferior hypophyseal arteries are the most common sources of vascular supply to PAs. ${ }^{24}$ This vascular network, especially the hypophyseal portal circulation with its fenestrated capillary network, may allow blood-borne circulating neoplastic cells entry into the PA.

Our case has several attributes that made the final diagnosis surprising and unusual. Only three prior cases have described a melanoma metastasis to a PA (Table 1). ${ }^{12,13,25}$ In all three cases, patients reported progressive vision loss, and pituitary apoplexy was suspected in two. Common presentations included hyperprolactinemia and headache. Our patient presented without headache or worsening endocrine abnormalities, although his history of pituitary axis dysfunction (without diabetes insipidus) may have suggested the presence of a metastasis over a solitary adenoma. ${ }^{4,9,20-22}$ Notably, the tumor demonstrated slow growth for 2 years after it was first discovered on MRI, and imaging characteristics supported the diagnosis of macroadenoma over metastasis, even in the setting of disseminated melanoma. Although pituitary metastases are often associated with rapid growth relative to PAs, ${ }^{26}$ in our patient it is possible the melanoma tissue had been growing and subsequently hemorrhaged (especially given the propensity for metastatic melanoma to hemorrhage), relative to the slower-growing adenoma component. The timing of the metastasis remains unknown, and a PA was almost certainly present for a period of time before metastasis of the melanoma and subsequent hemorrhage. Furthermore, the patient's only known intracranial metastasis was localized to the pituitary region. In clinical studies, $10 \%$ to $40 \%$ of malignant melanoma cases metastasize to the central nervous system ${ }^{27,28}$ often with multiple lesions. ${ }^{29}$ In a retrospective chart review of 80 patients diagnosed with metastatic melanoma with intracranial metastases, 39 (49\%) had single and 41 (51\%) had multiple metastases. $^{30}$

In total during his treatment course with the oncology department, our patient received ipilimumab, pembrolizumab, and ibrutinib therapy. Ipilimumab, a CTLA-4 inhibitor, and pembrolizumab, a programmed cell death-1 inhibitor, are immune checkpoint inhibitors. $^{31,32}$ Ibrutinib is an oral inhibitor of Bruton tyrosine kinase, which can cross the blood-brain barrier. ${ }^{31,33}$ In the treatment of most brain metastases, the blood-brain barrier poses an obstacle for treatment with chemotherapy and/or immunotherapy. ${ }^{34}$ In particular, immune checkpoint inhibitors are antibodies, which raises concerns over their ability to cross the blood-brain barrier. ${ }^{32}$ However, the pituitary is located outside of the blood-brain barrier, which may have both promoted tumor seeding and subsequently allowed therapy to access our patient's metastasis.

Differentiation on neuroimaging studies is key but can be challenging because of only subtle differences, as noted in our patient. The presence of melanin ${ }^{15}$ and melanoma's propensity for hemorrhage $^{35}$ give distinctive MRI characteristics that allow for better identification of the malignant cancer. Melanoma metastases were more likely to be hypointense on T2-weighted images and hyperintense on T1-weighted images. ${ }^{15}$ However, blood and fat also appear hyperintense on T1-weighted images, possibly interfering with diagnosis. $^{36}$ Macroadenomas most often appear heterogeneous and hyperintense on T2-weighted images and isointense on T1weighted images. ${ }^{37}$ These imaging findings were noted in our 
patient, further supporting the radiographic diagnosis of PA rather than metastatic melanoma initially. Although features such as hemorrhage and invasion into the cavernous sinus may help to distinguish a pituitary metastasis from an adenoma, these characteristics are not specific to metastasis. PAs commonly invade the cavernous sinus. ${ }^{38}$ Additionally, in a prospective study of patients with pituitary macroadenoma, almost half of patients $(45.45 \%)$ were noted to have imaging features of intratumoral hemorrhage. ${ }^{37}$ The level of variability in tumor presentations and imaging characteristics requires a final diagnosis to be made histopathologically.

Our case presents a rare example of collision tumor, but there are several limitations to address. Given the delayed referral to neurosurgery, our patient did not have consistent preoperative imaging, ophthalmology evaluation, and laboratory monitoring, which are otherwise standard of care for our pituitary patients. Further, postoperatively, the patient died of an unrelated cause and was unable to undergo follow-up imaging or additional oncology care. As a result, our evaluation and management of this patient were significantly limited. However, we believe that this unique case still offers valuable management lessons on early intervention and pathological diagnosis when imaging results may be inconclusive in a patient with metastatic disease and pituitary dysfunction.

\section{Lessons}

We describe a patient with diffusely disseminated melanoma who presented with hypopituitarism and intratumoral hemorrhage of a known sellar mass that, upon resection, was found to be melanoma metastasis to a PA. Our findings highlight the novel clinical and neuroimaging presentation of a rare collision tumor and the importance of pathological examination to determine the final diagnosis.

\section{References}

1. Abrams HL, Spiro R, Goldstein N. Metastases in carcinoma: analysis of 1000 autopsied cases. Cancer. 1950;3(1):74-85.

2. Saeger W, Lüdecke DK, Buchfelder M, et al. Pathohistological classification of pituitary tumors: 10 years of experience with the German Pituitary Tumor Registry. Eur J Endocrinol. 2007;156(2): 203-216.

3. Valassi E, Biller BMK, Klibanski A, Swearingen B. Clinical features of nonpituitary sellar lesions in a large surgical series. Clin Endocrinol (Oxf). 2010;73(6):798-807.

4. Fassett DR, Couldwell WT. Metastases to the pituitary gland. Neurosurg Focus. 2004;16(4):E8

5. McCutcheon IE, Waguespack SG, Fuller GN, Couldwell WT. Metastatic melanoma to the pituitary gland. Can J Neurol Sci. 2007;34(3):322-327.

6. Cox HH, Sloan LH. Melanoma: report of a case of apparently primary in the jejunum, the presenting symptoms resulting from metastasis in the hypophysis cerebri. J Am Med Assoc. 1924;82(25):2021-2025.

7. Mayr NA, Yuh WT, Muhonen MG, et al. Pituitary metastases: MR findings. J Comput Assist Tomogr. 1993;17(3):432-437.

8. Leung GKK, Chow WS, Tan KCB, et al. Metastatic melanoma of the pituitary gland. Case report. J Neurosurg. 2003;99(5): 913-915.

9. Guzel A, Maciaczyk J, Dohmen-Scheufler H, et al. Multiple intracranial melanoma metastases: case report and review of the literature. J Neurooncol. 2009;93(3):413-420.

10. Masui K, Yonezawa T, Shinji Y, et al. Pituitary apoplexy caused by hemorrhage from pituitary metastatic melanoma: case report. Neurol Med Chir (Tokyo). 2013;53(10):695-698.
11. Dasgupta T, Brasfield R. Metastatic melanoma. A clinicopathological study. Cancer. 1964;17:1323-1339.

12. Yang $C$, Liu $L$, Lan $X$, et al. Progressive visual disturbance and enlarging prolactinoma caused by melanoma metastasis: a case report and literature review. Medicine (Baltimore). 2017;96(14): e6483.

13. Ramos R, Machado M, Antunes $C$, et al. Metastasis of a dorsal melanoma to a pituitary adenoma mimicking pituitary apoplexy. Arq Bras Neurocir Braz Neurosurg. 2017;36(4):238-242.

14. Marx HF, Colletti PM, Raval JK, et al. Magnetic resonance imaging features in melanoma. Magn Reson Imaging. 1990;8(3): 223-229.

15. Gaviani P, Mullins ME, Braga TA, et al. Improved detection of metastatic melanoma by $2^{*}$-weighted imaging. AJNR Am J Neuroradiol. 2006;27(3):605-608.

16. Moody P, Murtagh K, Piduru S, et al. Tumor-to-tumor metastasis: pathology and neuroimaging considerations. Int J Clin Exp Pathol. 2012;5(4):367-373.

17. Schmitt HP. Metastases of malignant neoplasms to intracranial tumours: the "tumour-in-a-tumour" phenomenon. Virchows Arch A Pathol Anat Histopathol. 1984;405(1):155-160.

18. Bhargava P, McGrail KM, Manz HJ, Baidas S. Lung carcinoma presenting as metastasis to intracranial meningioma: case report and review of the literature. Am J Clin Oncol. 1999;22(2): 199-202.

19. Baratelli GM, Ciccaglioni B, Dainese E, Arnaboldi L. Metastasis of breast carcinoma to intracranial meningioma. J Neurosurg Sci. 2004;48(2):71-73.

20. Max MB, Deck MD, Rottenberg DA. Pituitary metastasis: incidence in cancer patients and clinical differentiation from pituitary adenoma. Neurology. 1981;31(8):998-1002.

21. Juneau $P$, Schoene WC, Black $P$. Malignant tumors in the pituitary gland. Arch Neurol. 1992;49(5):555-558.

22. Chiang MF, Brock M, Patt S. Pituitary metastases. Neurochirurgia (Stuttg). 1990;33(4):127-131.

23. Zacest AC, Besser M, Stevens G, et al. Surgical management of cerebral metastases from melanoma: outcome in 147 patients treated at a single institution over two decades. $J$ Neurosurg. 2002;96(3):552-558.

24. Powell DF, Baker HL Jr, Laws ER Jr. The primary angiographic findings in pituitary adenomas. Radiology. 1974;110(3):589-595

25. Jung SH, Hsu YY, Chuang CC, et al. Brain Pathology Case of the Month - July 2006. International Society of Neuropathology. Accessed June 6, 2020. https://path.upmc.edu/divisions/neuropath/ bpath/cases/case124.html

26. Dutta P, Bhansali A, Shah VN, et al. Pituitary metastasis as a presenting manifestation of silent systemic malignancy: a retrospective analysis of four cases. Indian J Endocrinol Metab. 2011;15(suppl 3): S242-S245.

27. Goulart CR, Mattei TA, Ramina R. Cerebral melanoma metastases: a critical review on diagnostic methods and therapeutic options. ISRN Surg. 2011;2011:276908.

28. Chiarion-Sileni V, Murr R, Pigozzo J, et al. Brain metastases from malignant melanoma. Forum (Genova). 2003;13(2):170-190.

29. Nayak L, Lee EQ, Wen PY. Epidemiology of brain metastases. Curr Oncol Rep. 2012;14(1):48-54.

30. Nussbaum ES, Djalilian HR, Cho KH, Hall WA. Brain metastases. Histology, multiplicity, surgery, and survival. Cancer. 1996;78(8): 1781-1788

31. Zukas AM, Schiff D. Neurological complications of new chemotherapy agents. Neuro Oncol. 2018;20(1):24-36.

32. Cohen JV, Tawbi H, Margolin KA, et al. Melanoma central nervous system metastases: current approaches, challenges, and opportunities. Pigment Cell Melanoma Res. 2016;29(6):627-642. 
33. Bernard S, Goldwirt L, Amorim S, et al. Activity of ibrutinib in mantle cell lymphoma patients with central nervous system relapse. Blood. 2015;126(14):1695-1698.

34. Kim M, Kizilbash SH, Laramy JK, et al. Barriers to effective drug treatment for brain metastases: a multifactorial problem in the delivery of precision medicine. Pharm Res. 2018;35(9): 177.

35. Ginaldi S, Wallace S, Shalen $\mathrm{P}$, et al. Cranial computed tomography of malignant melanoma. AJR Am J Roentgenol. 1981;136(1):145-149.

36. Chappell PM, Kelly WM, Ercius M. Primary sellar melanoma simulating hemorrhagic pituitary adenoma: MR and pathologic findings. AJNR Am J Neuroradiol. 1990;11(5):1054-1056.

37. Gupta K, Sahni S, Saggar K, Vashisht G. Evaluation of clinical and magnetic resonance imaging profile of pituitary macroadenoma: a prospective study. J Nat Sci Biol Med. 2018;9(1):34-38.

38. Knosp E, Steiner E, Kitz K, Matula C. Pituitary adenomas with invasion of the cavernous sinus space: a magnetic resonance imaging classification compared with surgical findings. Neurosurgery. 1993;33(4):610-618.

\section{Disclosures}

The authors report no conflict of interest concerning the materials or methods used in this study or the findings specified in this paper.

\section{Author Contributions}

Conception and design: Zada, Rangwala, Kammen, Kramer, Rutkowski. Acquisition of data: Zada, Rangwala, Kammen. Analysis and interpretation of data: Zada, Lamorie-Foote, Rangwala, Kammen, Gnass, Rutkowski, Strickland. Drafting the article: Lamorie-Foote, Rangwala, Kammen, Rutkowski, Strickland. Critically revising the article: Zada, Lamorie-Foote, Rangwala, Kammen, Kramer, Rutkowski, Strickland, Carmichael. Reviewed submitted version of manuscript: Lamorie-Foote, Rangwala, Kammen, Kramer, Strickland, Carmichael. Approved the final version of the manuscript on behalf of all authors: Zada. Administrative/technical/material support: Zada, Rangwala. Study supervision: Zada, Rangwala, Rutkowski.

\section{Correspondence}

Gabriel Zada: Keck School of Medicine, University of Southern California, Los Angeles, CA. gabriel.zada@med.usc.edu. 